\title{
Psychiatric Symptoms in a Patient with Influenza A (H1N1) Treated with Oseltamivir (Tamiflu): A Case Report
}

\author{
Sang Won Jeon, Changsu Han \\ Department of Psychiatry, Korea University Ansan Hospital, Korea University College of Medicine, Ansan, Korea
}

Oseltamivir is the most common antiviral drug used to treat and prevent influenza. Epidemiological studies performed in Japan, the United States, and the United Kingdom indicate that oseltamivir may cause psychiatric symptoms; however, the underlying mechanism has not been elucidated. In South Korea, interest in oseltamivir has increased with the spread of the new influenza virus; however, no case report or investigation of psychiatric symptoms associated with the drug has been reported to date. Here, we report a case of a 22-year-old male who complained of mood swings, suicidal feelings, auditory hallucinations, memory deterioration, and insomnia after taking oseltamivir.

KEY WORDS: Oseltamivir; Tamiflu; Human influenza; Psychiatric symptom; Side effect.

\section{INTRODUCTION}

Oseltamivir (Tamiflu; F. Hoffmann-La Roche, Ltd., Basel, Switzerland) is the most frequently used antiviral agent for the treatment and prevention of influenza, and its use has increased since the influenza A (H1N1) epidemic. ${ }^{1,2)}$ With the increased use of oseltamivir, psychiatric symptoms have been reported as side effects. Between 1999 and 2007, a total of 480 million patients worldwide were administered oseltamivir, and psychiatric side effect surveys were conducted in Japan $(n=2,772)$, the United States $(n=190)$, and other countries $(n=89) .{ }^{3)}$ Psychiatric side effects were more common in infants and children aged 16 years or younger than in adults $(2,218$ children vs. 833 adults), ${ }^{4)}$ and generally occurred within 48 hours of receiving oseltamivir. ${ }^{5,6)}$ According to the International Classification of Diseases 10th revision (ICD-10), the major symptoms include abnormal behavior $(1,160$ events, 38\%), delusions/perceptual disturbances (661 events, $21.7 \%$ ), and delirium or delirium-like events. ${ }^{3-7)}$ These side effects may lead to accidents, injuries, or suicides.

\footnotetext{
Received: December 9, 2014 / Revised: December 30, 2014

Accepted: December 31, 2014

Address for correspondence: Changsu Han, MD, PhD

Department of Psychiatry, Korea University Ansan Hospital, 123

Jeokgeum-ro, Danwon-gu, Ansan 15355, Korea

Tel: +82-31-412-4931, Fax: +82-31-412-5144

E-mail: hancs@korea.ac.kr
}

Unlike research abroad, few studies of the psychiatric side effects of oseltamivir have been conducted in South Korea. Moreover, despite various epidemiological and experimental studies, few studies have investigated the entire disease cycle from symptom onset and treatment through follow up. It is likely that the extensive use of oseltamivir to treat influenza will continue into the future; thus, it is important that attention be given to the psychiatric side effects of the drug.

\section{CASE}

A 22-year-old male visited our psychiatry clinic with the major complaints of mood swings, suicidal impulses, auditory hallucinations, memory deterioration, and insomnia, which had occurred several times a day for the past 5 days. He had no personal or familial psychiatric history. He was well adjusted and had no problems with his family or other relationships. Twelve days earlier, the patient had developed fever and respiratory symptoms suspected to be indicative of influenza A (H1N1). Ten days before his visit, he was prescribed $75 \mathrm{mg}$ oseltamivir (Tamiflu) and $650 \mathrm{mg}$ acetaminophen (Tylenol ER; Janssen Korea, Ltd., Seoul, Korea), which he took in the morning and evening daily for 5 days. Nine days before his visit, the diagnosis was confirmed by an influenza A (H1N1) polymerase chain reaction (PCR) test, and he continued to take Tamiflu as scheduled. During the 5 days that

(c) This is an Open-Access article distributed under the terms of the Creative Commons Attribution Non-Commercial License (http://creativecommons.org/licenses/by-nc/4.0) which permits unrestricted non-commercial use, distribution, and reproduction in any medium, provided the original work is properly cited. 
he was taking Tamiflu, the patient continued his normal routine, but felt a slight deterioration in mood and memory and suffered from insomnia. The patient's fever and respiratory symptoms were in remission 3 days after the initiation of Tamiflu; however, the deterioration in his mood and memory and the insomnia did not improve significantly. The next day, when he finished the 5-day course of Tamiflu, he started to develop significant psychiatric symptoms. He experienced manic symptoms for periods ranging from 10 minutes to 2 hours and then abruptly felt depressed and burst into tears with frequent nihilistic and sin delusions. Two days after he finished taking Tamiflu and 3 days before his visit, the patient's mood swings and instability grew worse, and he experienced auditory hallucinations similar to a telephone conversation between a man and a woman and text message alarm ringtones for mobile phones. Because of his memory deterioration, the patient readily forgot what he had done several hours or days earlier. He was not able to sleep more than 2-3 hours a day. Eventually, the patient visited our psychiatric clinic and was admitted under the suspicion of bipolar disorder, an organic mental disorder such as delirium and encephalitis, and psychotic disorders caused by his drug intake.

The patient was admitted to the closed ward and treated with antipsychotic medication by $10 \mathrm{mg}$ intramuscular olanzapine (Zyprexa; Eli Lilly and Company, Ltd., Indianapolis, IN, USA) and $10 \mathrm{mg}$ oral olanzapine. After drug treatment, the patient was able to sleep for 9 hours. The next morning, his suicidal ideas had disappeared, his mood swings decreased, and the frequency and intensity of his auditory hallucination were reduced significantly.

Single-photon emission computed tomography (SPECT) and magnetic resonance imaging (MRI) scans performed the second day after the patient was admitted to hospital yielded normal findings with no deterioration in brain function or activity and no changes in brain structure. The electroencephalogram (EEG) showed no abnormal brainwave patterns, and the neurological examinations revealed no abnormal findings. Physical examinations (blood test, serum electrolyte, blood glucose, blood urea nitrogen, creatinine, liver function test, serum syphilis, HIV test, thyroid function test, serum vitamin B12, folate), chest X-ray, electrocardiogram, and urine tests all showed normal results. The patient did not have a history of drug use, and the result of his urine drug-screening test was negative. The influenza A (H1N1) PCR test was repeated, and a negative result was confirmed. The patient received $5 \mathrm{mg}$ olanzapine orally on day 2 following ad- mission to the hospital, after which he showed a normal sleep pattern.

From day 3 after admission, the patient's mood and behaviors became normal, and no auditory hallucinations or memory deterioration were observed. Given the rapid improvement in his symptoms, antipsychotic medication was ceased. Psychiatric observation, which was continued until day 8 , revealed no specific psychiatric symptoms. He talked about his symptoms with an ego-dystonic attitude and a high insight level. On day 8 after admission, the patient was discharged based on his normal test findings and psychiatric observation results. No drug prescription was made. The discharge diagnosis was 'mental and behavioral disorder due to the use of other psychoactive substances' (F19.8; ICD-10).

He was followed up 2 weeks after discharge, at which point, he had adjusted to his normal daily routine. The absence of symptoms was confirmed at the next follow-up 4 weeks after discharge. At 10 months after discharge, the patient successfully managed his daily life with no psychiatric symptoms.

\section{DISCUSSION}

We describe a patient with psychiatric symptoms that resulted from oseltamivir intake following an influenza A (H1N1) infection. Several factors pointed toward oseltamivir as the cause of the patient's psychiatric symptoms. First, involvement of other psychiatric conditions was ruled out by psychiatric interviews and assessments, and the presence of organic mental conditions and psychotic disorders caused by the intake of other medications was excluded through physical examinations, brain imaging scans, and EEG. Second, the patient's symptoms were similar to those reported in previous studies of psychiatric symptoms associated with oseltamivir. Third, the patient was highly responsive to antipsychotic medications and fully recovered in a short period of time, which suggested drug-induced psychosis. Fourth, because the patient was unaware of the side effects of oseltamivir and the possibility of developing psychiatric symptoms, he did not have a bias or a sensitive response to the drug. Fifth, the patient's symptoms were confirmed to have developed independently during the time of the medication intake, and he showed no symptoms during a 10-month follows-up period after the reversal of his symptoms.

However, our patient showed some differences from previously described cases. It has been known that psychiatric symptoms usually develop within 48 hours of oselta- 
mivir intake ${ }^{5,6)}$; however, in our case, the psychiatric symptoms became obvious 5 days after oseltamivir intake.

The mechanism underlying oseltamivir-induced psychiatric symptoms is not well understood. One hypothesis is that a neuraminidase inhibitor that has antiviral effects crosses the blood-brain barrier (BBB) into the central nervous system. ${ }^{8)}$ Studies in mice have shown that although oseltamivir carboxylate, an oseltamivir metabolite, does not pass through the $\mathrm{BBB}$ readily, it may do so when combined with other agents or when the BBB is damaged. ${ }^{9)}$ Moreover, oseltamivir carboxylate has been shown to cause neuronal excitability. ${ }^{10)}$ A previous study found that plasma and brain oseltamivir carboxylate concentrations were higher in young mice than in adults, suggesting that oseltamivir is more likely to have adverse effects in infants and adolescents than in adults. ${ }^{11)}$

Because the effect of the influenza virus on the central nervous system is not fully understood, we are not able to conclude whether the symptoms experienced by our patient were consistent with the clinical course of influenza. Moreover, the symptoms may have been the result of immunological rather than influenza viral effects on the central nervous system. ${ }^{8,9)}$ Complications of influenza, such as encephalitis and encephalopathy, can be diagnosed by neurological examinations and brain imaging. However, these techniques are not sufficient for the diagnosis of central nervous system complications not caused by the direct invasion of the influenza virus, such as immunological or unknown mechanisms. ${ }^{12)}$

Oseltamivir is generally prescribed in combination with nonsteroidal anti-inflammatory drugs (NSAIDs) or antihistamine agents. The effect of interactions between oseltamivir and these agents on the central nervous system is poorly understood. The oseltamivir metabolite changes as it interacts with other drugs and may affect the central nervous system. ${ }^{9,10)}$

Psychiatric side effects of oseltamivir have been reported more frequently in Japan than in the United States and Europe. ${ }^{3)}$ This may be explained by the extensive use of oseltamivir during the influenza pandemic in Japan; however, genetic features associated with ethnicity or geographic factors may also contribute to the higher in- cidence of psychiatric symptoms.

Although several studies have demonstrated that oseltamivir induces psychiatric symptoms, this issue remains under debate. Further study of the mechanisms underlying the psychiatric side effects of the drug is needed. In the meantime, close observation and follow-up are recommended when oseltamivir is administered for the treatment of influenza.

\section{REFERENCES}

1. National Institute for Clinical Excellence. Guidance on the use of zanamivir, olsetamivir and amantadine for the treatment of influenza: Technology appraisal guidance. 4th ed. Washington, DC:National Institute for Clinical Excellence;2010.

2. Turner D, Wailoo A, Nicholson K, Cooper N, Sutton A, Abrams K. Systematic review and economic decision modeling for the prevention and treatment of influenza $A$ and B. Health Technol Assess 2003;7:iii-iv, xi-xiii, 1-170.

3. Toovey S, Rayner C, Prinssen E, Chu T, Donner B, Thakrar $\mathrm{B}$, et al. Assessment of neuropsychiatric adverse events in influenza patients treated with oseltamivir: a comprehensive review. Drug Saf 2008;31:1097-1114.

4. Maxwell SR. Tamiflu and neuropsychiatric disturbance in adolescents. BMJ 2007;334:1232-1233.

5. Fuyuno I. Tamiflu side effects come under scrutiny. Nature 2007;446:358-359.

6. US Food and Drug Administration. Safety alerts for drugs, biologist, medical devices, and dietary supplements. The FDA safety information and adverse event reporting program. 12th ed. New York:US Food and Drug Administration;2011.

7. US Ministry of Health. Working group for clinical investigation for oseltamivir phosphate. 1st ed. Boston:US Ministry of Health;2007.

8. Matheson NJ, Harnden AR, Perera R, Sheikh A, SymmondsAbrahams M. Neuraminidase inhibitors for preventing and treating influenza in children. Cochrane Database Syst Rev 2007; (1):CD002744.

9. Izumi Y, Tokuda K, O’Dell K, Zorumski C, Narahashi T. Neuroexcitatory actions of Tamiflu and its carboxylate metabolite. Neurosci Let 2007;426:54-58.

10. Satoh K, Nonaka R, Ogata A, Nakae D, Uhehara S. Effects of oseltamivir phosphate (Tamiflu) and its metabolite (GS4071) on monoamine neurotransmission in the rat brain. Biol Pharm Bull 2007;30:1816-1818.

11. Ose A, Kusuhara H, Yamatsugu K, Kanai M, Shibasaki M, Fujita $\mathrm{T}$, et al. P-glycoprotein restricts the penetration of oseltamivir across the blood-brain barrier. Drug Metab Dispos 2008;36:427-434.

12. Hama R. Fatal neuropsychiatric adverse reactions to oseltamivir: Case series and overview of casual relationships. Int J Risk Saf Med 2008;20:5-36. 